\title{
BCG vaccine protection from severe coronavirus disease 2019 (COVID-19)
}

\author{
Luis E. Escobar ${ }^{a, 1}$, Alvaro Molina-Cruz ${ }^{b}{ }^{\infty}$, and Carolina Barillas-Mury ${ }^{b, 1}$ \\ ${ }^{a}$ Department of Fish and Wildlife Conservation, Virginia Polytechnic Institute and State University, Blacksburg, VA 24601 ; and ${ }^{b}$ Laboratory of Malaria and \\ Vector Research, National Institute of Allergy and Infectious Diseases, National Institutes of Health, Bethesda, MD 20892
}

Contributed by Carolina Barillas-Mury, June 9, 2020 (sent for review May 1, 2020; reviewed by Serap Aksoy and Rita R. Colwell)

\begin{abstract}
A series of epidemiological explorations has suggested a negative association between national bacillus Calmette-Guérin (BCG) vaccination policy and the prevalence and mortality of coronavirus disease 2019 (COVID-19). However, these comparisons are difficult to validate due to broad differences between countries such as socioeconomic status, demographic structure, rural vs. urban settings, time of arrival of the pandemic, number of diagnostic tests and criteria for testing, and national control strategies to limit the spread of COVID-19. We review evidence for a potential biological basis of BCG cross-protection from severe COVID-19, and refine the epidemiological analysis to mitigate effects of potentially confounding factors (e.g., stage of the COVID-19 epidemic, development, rurality, population density, and age structure). A strong correlation between the BCG index, an estimation of the degree of universal BCG vaccination deployment in a country, and COVID19 mortality in different socially similar European countries was observed $\left(r^{2}=0.88 ; P=8 \times 10^{-7}\right)$, indicating that every $10 \%$ increase in the BCG index was associated with a $10.4 \%$ reduction in COVID-19 mortality. Results fail to confirm the null hypothesis of no association between BCG vaccination and COVID-19 mortality, and suggest that BCG could have a protective effect. Nevertheless, the analyses are restricted to coarse-scale signals and should be considered with caution. BCG vaccination clinical trials are required to corroborate the patterns detected here, and to establish causality between BCG vaccination and protection from severe COVID19. Public health implications of a plausible BCG cross-protection from severe COVID-19 are discussed.
\end{abstract}

BCG vaccination policy | COVID-19 coronavirus | cross-protection | mortality | pandemic

$\mathbf{T}^{\mathrm{T}}$ he bacillus Calmette-Guérin (BCG) vaccine, an attenuated Mycobacterium bovis (1), has been extensively used in national vaccination programs, as it confers cross-protection from Mycobacterium tuberculosis infection (2). BCG vaccination of newborns and infants prevents disseminated childhood tuberculosis disease (TB) and reduces the risk of pulmonary TB by about $50 \%$ (3). Even though the BCG vaccine has been in use for more than $90 \mathrm{y}$, with proven safety, its efficacy is still controversial (4). BCG vaccination has shown clear protection in children, but, in adults, its effects have been inconsistent (5). Many countries initiated national BCG vaccination in the middle of the twentieth century with variable levels of coverage using different BCG strains (6), number of doses, and delivery method (7). As the prevalence of TB decreased, countries like France, Germany, and Spain stopped mass vaccination of children and moved to vaccinate only individuals at high risk. Other countries like Russia, Ukraine, and China have continued national BCG vaccination to date. Some countries never established national universal BCG vaccination, including the United States and Italy, and only target high-risk individuals (7).

There is ample epidemiological evidence that BCG vaccination has broad protective effects that are not specific to $M$. tuberculosis infection. For example, in 1927, Swedish children who received $\mathrm{BCG}$ vaccination at birth had a mortality rate almost threefold lower than unvaccinated children. This decrease of mortality could not be explained by TB infection, and thus, early on, it was suggested that the very low mortality among BCGvaccinated children may be caused by nonspecific immunity (8). In West Africa, a BCG vaccination scar and a positive tuberculin reaction were associated with better survival during early childhood in an area with high mortality; this was not observed with other childhood vaccines (9). A general reduction in neonatal mortality linked to BCG vaccination was also reported in children from Guinea-Bissau, mainly due to fewer cases of neonatal sepsis, respiratory infection, and fever (10). The BCG vaccine appears to confer broad enhanced immunity to respiratory infections, as infants from Guinea-Bissau with acute viral infections of the lower respiratory tract were more likely to not have received BCG vaccination than matched controls (11). In Spain, hospitalizations due to respiratory infections in 0- to 14-y-old children not attributable to TB were significantly lower in BCG-vaccinated compared to non-BCG-vaccinated children (12). The observation that this protection was still present in 14-y-old children suggests that the broad protective effect of BCG can be long lasting. Taken together, our current understanding of broad immune protection mediated by trained immunity and the epidemiologic evidence of long-lasting protection from viral infections of the respiratory tract, conferred by BCG vaccination, offer a rational biological basis for the potential protective effect of BCG vaccination from severe coronavirus disease 2019 (COVID-19) (reviewed recently in ref. 13).

\section{Significance}

The COVID-19 pandemic is one of the most devastating in recent history. The bacillus Calmette-Guérin (BCG) vaccine against tuberculosis also confers broad protection against other infectious diseases, and it has been proposed that it could reduce the severity of COVID-19. This epidemiological study assessed the global linkage between BCG vaccination and COVID-19 mortality. Signals of BCG vaccination effect on COVID-19 mortality are influenced by social, economic, and demographic differences between countries. After mitigating multiple confounding factors, several significant associations between BCG vaccination and reduced COVID-19 deaths were observed. This study highlights the need for mechanistic studies behind the effect of BCG vaccination on COVID-19, and for clinical evaluation of the effectiveness of BCG vaccination to protect from severe COVID-19.

Author contributions: L.E.E., A.M.-C., and C.B.-M. designed research; L.E.E., A.M.-C., and C.B.-M. performed research; L.E.E. and A.M.-C. contributed new reagents/analytic tools; L.E.E., A.M.-C., and C.B.-M. analyzed data; L.E.E. and C.B.-M. wrote the paper; and A.M.-C. compiled epidemiological information from many different sources.

Reviewers: S.A., Yale University; and R.R.C., University of Maryland, College Park.

The authors declare no competing interest.

This open access article is distributed under Creative Commons Attribution License 4.0 (CC BY).

${ }^{1}$ To whom correspondence may be addressed. Email: escobar1@vt.edu or cbarillas@niaid. nih.gov.

This article contains supporting information online at https://www.pnas.org/lookup/suppl/ doi:10.1073/pnas.2008410117///DCSupplemental. 
Considering the cross-protection reported for BCG vaccination on viral respiratory infections, recent publications have proposed that BCG vaccination could have protective effects against COVID-19 infection (14-16). These publications, however, do not include extensive statistical analysis, and the World Health Organization has cautioned about the lack of research regarding BCG vaccination against COVID-19 infection (17). In view of the growing interest in assessing the plausible association between BCG vaccination and protection from severe COVID19, we assessed available global data on BCG and COVID-19 to investigate the hypothesis that countries without a national BCG vaccination program would have greater COVID-19 mortality than countries that have a program. We attempted to control for potential confounding variables among countries, such as level of urbanization, population density, age classes, access to health, income, education, and stage and size of the COVID-19 epidemic.

\section{Methods}

Broad Analysis. We collected COVID-19 mortality data by country, considering that numbers of deaths may be more reliable than the numbers of cases, since case numbers are greatly influenced by the intensity of diagnostic efforts, criteria for testing, number of asymptomatic cases, and availability and sensitivity of diagnostic tests (18). Number of deaths is also a good proxy of epidemic size during explosive epidemics, considering that, often, the number of cases is underreported due to overwhelmed and unprepared health systems (i.e., surveillance fatigue) (19). Although media reports suggest consistent underreporting of COVID-19 deaths globally (20), this parameter is harder to manipulate than case numbers. We used ANOVA and $t$ tests to assess effect of BCG vaccination policy on COVID-19 mortality, and used linear regressions $(21,22)$ to assess linkages between use of BCG vaccine and number of COVID-19 deaths $(\alpha=0.05)$. BCG vaccination data were collected at the country level based on policy of vaccination (i.e., current, interrupted, never) $(7,23,24)$ and the mean and median percentage of vaccination coverage during the period 1980-2018 (6), assigning zero coverage to countries where BCG national vaccination campaigns have never been conducted. COVID-19-related deaths were collected until April 22, 2020, and were standardized by population and stage of the epidemic (25). Independent (i.e., with vs. without BCG vaccination, policy of BCG vaccination in place, and percentage of vaccine coverage) and dependent variables (i.e., mean, median, and maximum deaths per million) were compared using data at specific times of each country's epidemic since the first death (e.g., 21 and $30 \mathrm{~d}$ since first death; days since first death until 0.1 and 1 death per million; middle point and full period since first death). This design allowed fair comparisons between countries at different epidemic stages and of different population sizes, accounting for incubation period (26).

Refined Analysis. The hypothesis that countries without a national BCG vaccination program would have greater COVID-19 mortality in adult populations than countries that have a program was investigated at the global level and filtered by social conditions. Analyses were performed initially for countries with BCG vaccination data, $\geq 1$ million inhabitants, and nonzero COVID-19-related deaths. We focused on social variables as potential confounding factors, excluding climatic conditions in view of the lack of evidence for temperature dependence of the COVID-19 epidemic $(27,28)$. Potential confounding variables were assessed based on access to health and education services and income (i.e., Human Development Index [HDI]) (29), population size (30), human density (31), urbanization (32), and age structure of the population (30). For a fair comparison between countries, and after a preliminary evaluation of association (SI Appendix, Table S1), BCG vaccination and COVID-19 deaths assessments were conducted for countries with at least one death per million inhabitants, $\geq 15 \%$ of population with an age of 65 y or more, $>60 \%$ of population living in urban areas, $<300$ inhabitants per square kilometer, and an HDI of $>0.7$. These inclusion criteria mitigated effects of confounding factors by comparing countries with similar social conditions (SI Appendix, Table S1). To explore variables' predictability, we developed multiple linear regression models between COVID19 deaths accumulated during the first month of mortalities by country (log) and the potential confounding variables and BCG data. We used adjusted Bayesian information criterion, adjusted $r^{2}$, and Mallow's $C p$ metrics to determine the best variable combination and select the optimal model for COVID-19 death estimation. Analyses included all predictor variables combinations and were performed in R software using the leaps package (21).
Considering the size and social disparities of the United States, BCG effect analyses were also performed considering the United States as a country, and separately by state.

At the regional level, we assessed the COVID-19 pandemic in the Americas assuming virus invasion by air traffic, as the pandemic presumably arrived in the American continent from Europe or Asia through this route. Air traffic has revealed a strong linear correlation between international COVID-19 cases and international passenger volume $\left(r^{2}=0.98, P<0.01\right)$ (33). In large countries, such as the United States, Mexico, and Brazil, an epidemiological analysis at a country level does not consider the intense clustering of cases in states with large metropolitan areas and international airports, that were focal points of the pandemic. Thus, we decided to compare COVID-19 mortality in US states without BCG vaccination that have a high number of confirmed cases (more than 20,000 by April 20,2020) with those states that were the main points of entry in Mexico and Brazil, as these two countries have current BCG vaccination programs. Evaluations were restricted to mortality $25 \mathrm{~d}$ after the first COVID-19 related death was registered. At the local level, we assessed the COVID-19 pandemic in Germany considering that East and West Germany followed different BCG vaccination schemes before the reunification in 1990. In West Germany, infants were vaccinated between 1961 and 1998 (7). In East Germany, infants and 15-y-old teenagers with a negative skin test were vaccinated from 1951 to 1975 with at least one dose of BCG (7). German states were compared accounting by age structure (34), considering that most of the mortality in Germany (95\%) was observed in older individuals (60 y old or more) (35). We explored whether the broader range in BCG vaccination in eastern states of older individuals could reduce the mortality from COVID-19.

\section{Results}

Coarse Analysis. Global analysis of COVID-19 mortality revealed heterogeneity of mortality among countries (Fig. $1 A$ ). Three variables, HDI, percentage of population $>65 \mathrm{y}$ age, and urbanization, were consistently and positively associated with COVID-19 mortality (SI Appendix, Fig. S1), with the strongest association between HDI and COVID-19 mortality (Fig. $1 B$ and SI Appendix, Fig. S1). The global trend of vaccination policy (Fig. $1 A$ and $S I$ Appendix, Fig. S2) suggested an inverse association between BCG vaccination policy and COVID-19 mortality (Fig. $1 C$ and SI $A p$ pendix, Fig. S3). Coarse analysis revealed a consistent link between BCG vaccination and COVID-19 mortality under different scenarios of data curation (SI Appendix, Tables S2-S4). For example, countries with a stronger BCG vaccination policy had significantly lower COVID-19 deaths/million (1 million) (SI Appendix, Table $\mathrm{S} 2$ and Fig. $2 A$ ). More broadly, countries with current BCG vaccination had lower deaths as compared to countries with lack of, or interrupted, BCG vaccination (SI Appendix, Table S3). Similarly, the percentage of BCG coverage was negatively associated with COVID-19 deaths per million (SI Appendix, Table S4). The association was consistently supported by the coarse-level analysis when considering the United States as a country or by analyzing individual states, and when correcting by the stage of the epidemic (SI Appendix, Tables S2-S4).

Filtered Analysis. When the effects of potentially confounding factors on the dependent variables were mitigated by including only countries with at least one death per million inhabitants, $\geq 15 \%$ of population with an age of $65 \mathrm{y}$ or more, $>60 \%$ of population living in urban areas, $<300$ inhabitants per square kilometer, and an HDI of $>0.7$ (SI Appendix, Table S1), we were left with 22 socially similar countries. The control of confounding variables reduced the overall significant effect and association between BCG and COVID-19 deaths (i.e., amount of significant evaluations for linear models went from $46 \%$ for coarse analyses vs. $19 \%$ for refined; ANOVA: coarse $=100 \%$ vs. refined $=31 \%$; $t$ test: coarse $=92 \%$ vs. refined $=37 \%$; SI Appendix, Tables S2-S4) Nevertheless, a significant association and effect was still detected for several comparisons controlling for social conditions and stage of the epidemic by country (SI Appendix, Tables S2-S4). For example, type of BCG policy (i.e., current, interrupted, and never) had a significant effect on COVID-19 deaths per million (Fig. $2 B$ 

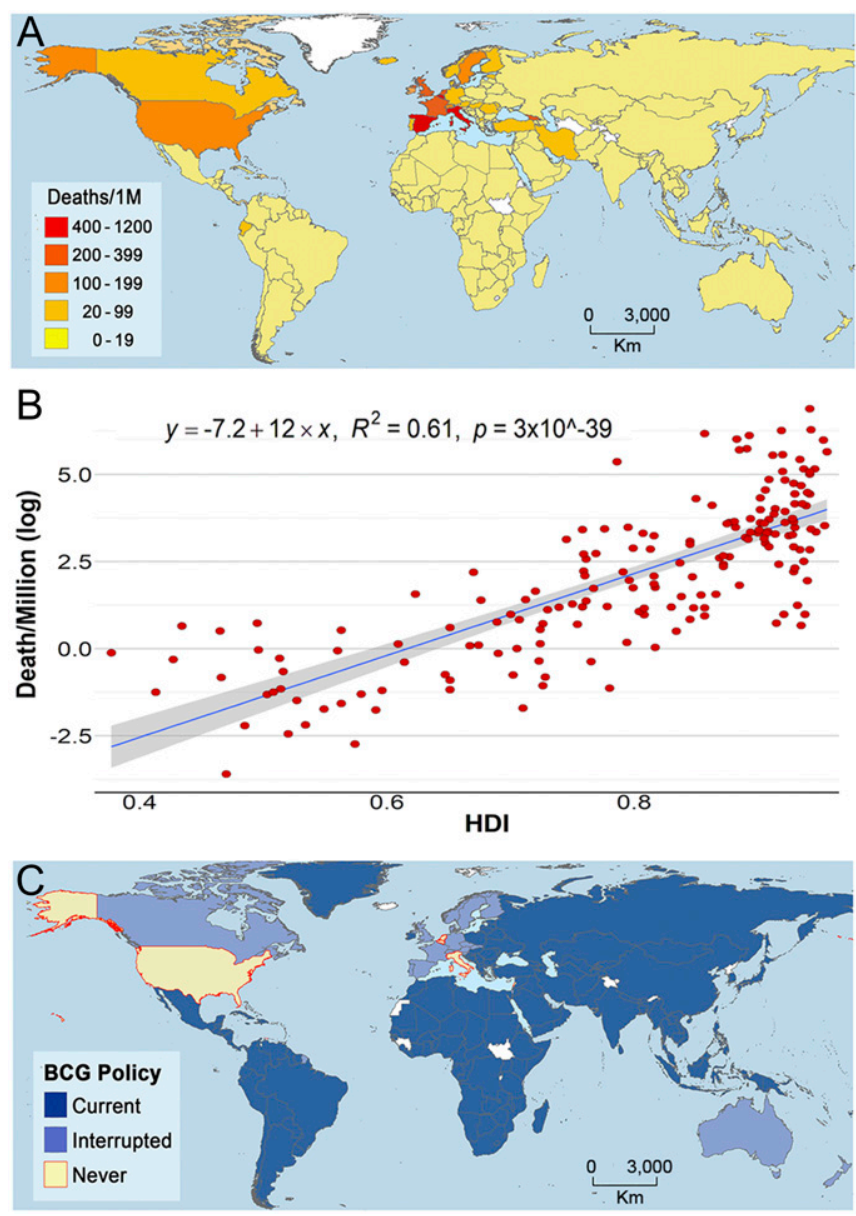

Fig. 1. COVID-19 mortality, human development, and BCG vaccination policy by country. (A) Map showing the COVID-19 mortality per million inhabitants in countries worldwide. COVID-19-related deaths per country per million inhabitants denoting countries with low (yellow) to high (red) mortality. (B) COVID-19 mortality per million inhabitants (log) vs. HDI in different countries worldwide. United States data appear by state. (C) Map showing the BCG vaccination policy in countries that currently have universal BCG vaccination program (Current), countries with interrupted BCG vaccination programs (Interrupted), and countries that never implemented a universal vaccination program (Never). Countries without information appear in white.

and SI Appendix, Table S2), with a stronger policy being associated with lower COVD-19 mortality. Additionally, countries with current vaccination had lower deaths as compared with countries with lack of or interrupted BCG vaccination (Fig. $2 C$ and SI Appendix, Table S3). Similarly, mean BCG coverage (percent) was negatively associated with COVID-19 deaths per million reported by country during the first month of the pandemic (Fig. 2D and SI Appendix, Table S4). Our multivariate model revealed that HDI and population density had the best predictive capacity of COVID-19 deaths $\left(r^{2}=0.49, F[2,113]=55.57, P<0.001\right)($ SI Appendix, Fig. $\mathrm{S} 4 A-C)$. It also showed that some of the variables evaluated were correlated with each other. For example, the percentage of population $>65 \mathrm{y}$ of age and HDI are strongly correlated, while there is a moderate correlation between the time period of universal BCG vaccination and population density (SI Appendix, Fig. $\mathrm{S} 4 D)$.

Arrival of the Pandemic to the Americas. The COVID-19 pandemic arrived in the Americas by air traffic, and regions with a high number of international flights from Europe have been the most affected. Thus, we decided to compare COVID-19 mortality in states from the United States without BCG vaccination with those that were the main points of entry in Mexico and Brazil, as these two countries have current BCG vaccination programs. We found that COVID-19 mortality in the states of New York, Illinois, Louisiana, Alabama, and Florida (unvaccinated) was significantly higher $(t$ [237] $=14.274, P<0.001)$ than states from BCG-vaccinated countries (Pernambuco, Rio de Janeiro, and Sao Paulo in Brazil; Mexico State and Mexico City in Mexico) (Fig. 3 A, Left). This is remarkable, considering that three states from Latin America have much higher population densities than the North American states analyzed, including New York (Fig. 3 A, Right).

The Pandemic in Europe. We also found broad differences in COVID-19 mortality within Europe. Germany provides a unique opportunity to compare the potential effect of age of BCG vaccination on susceptibility to COVID-19, as, before the unification, East and West Germany followed different vaccination schemes. In West Germany, those 22 y to 59 y old today were vaccinated, while, in East Germany, those 45 y to 84 y old today received at least one dose of BCG (Fig. $3 B$ and $C$ ). A comparison of these two regions revealed that the average COVID19 mortality rate in western German states (40.5 per million) was 2.9-fold higher than in eastern states (14.2 per million) (Fig. 3D). Similarly, the mean mortality in Western Europe was 9.92 times higher than in Eastern Europe $(t[11]=-2.592, P<0.025)$ (Fig. $4 A$ ), where countries, in general, have active universal BCG vaccination programs (Fig. $4 B$ ).

Western Europe has a complex history of BCG vaccination, ranging from Finland, which had a universal vaccination program for $65 \mathrm{y}$ initiated in 1941, to other countries like Italy, Belgium, and The Netherlands, which have never had universal BCG vaccination (Fig. $4 B$ ). To give these differences a quantitative value, a BCG vaccination index was developed by multiplying the age of the older group that was vaccinated (because COVID-19 mortality increases by age) by the number of years that a program was maintained. To adjust the index to a scale from 0 to 1 , values were divided by a parameter of the maximum score expected $(75 \times 75=5,625$, based on countries with $75 \mathrm{y}$ of uninterrupted vaccination; Fig. $4 C)$. We found a significant $\left(r^{2}=\right.$ $0.49, P<0.00001)$ negative correlation between BCG index and COVID-19 mortality in European countries that administer the BCG vaccine to infants by intradermal injection (Fig. $4 D$ ). In this analysis, the United Kingdom was excluded because it administered the vaccine to older children ( 12 y to $13 \mathrm{y}$ old) (7), and France was excluded because it administered the vaccine to infants and young children going to daycare, or to children of school age (36). We noted that COVID-19 mortality was higher in the United Kingdom and France compared to Germany, Scandinavia, or Eastern European countries (Fig. 4A). Finally, to try to control for other confounding factors that could affect COVID-19 mortality, we repeated the analysis including only those European countries that are socially similar (see Filtered Analysis) and normalized the time of arrival of the pandemic by comparing the mortality during the first $30 \mathrm{~d}$ after the first COVID-19 death was reported in each country. A highly significant linear correlation was found between the BCG index and mortality during the first month of the pandemic $\left(r^{2}=0.88 ; P=\right.$ $8 \times 10^{-7}$ ) (Fig. $4 E$ ); indicating that every $10 \%$ increase in the BCG index is associated with a $10.4 \%$ reduction in COVID-19 mortality.

\section{Discussion}

Classic Pathogen-Specific Immunity vs. Broad Protection through Innate Immune Training. Cellular immunity is very important to control infections by intracellular pathogens, such as $M$. tuberculosis, responsible for TB in humans (37). Gamma IFN $(\gamma$-IFN) is a key cytokine produced by CD4+ T cells that mediates macrophage 

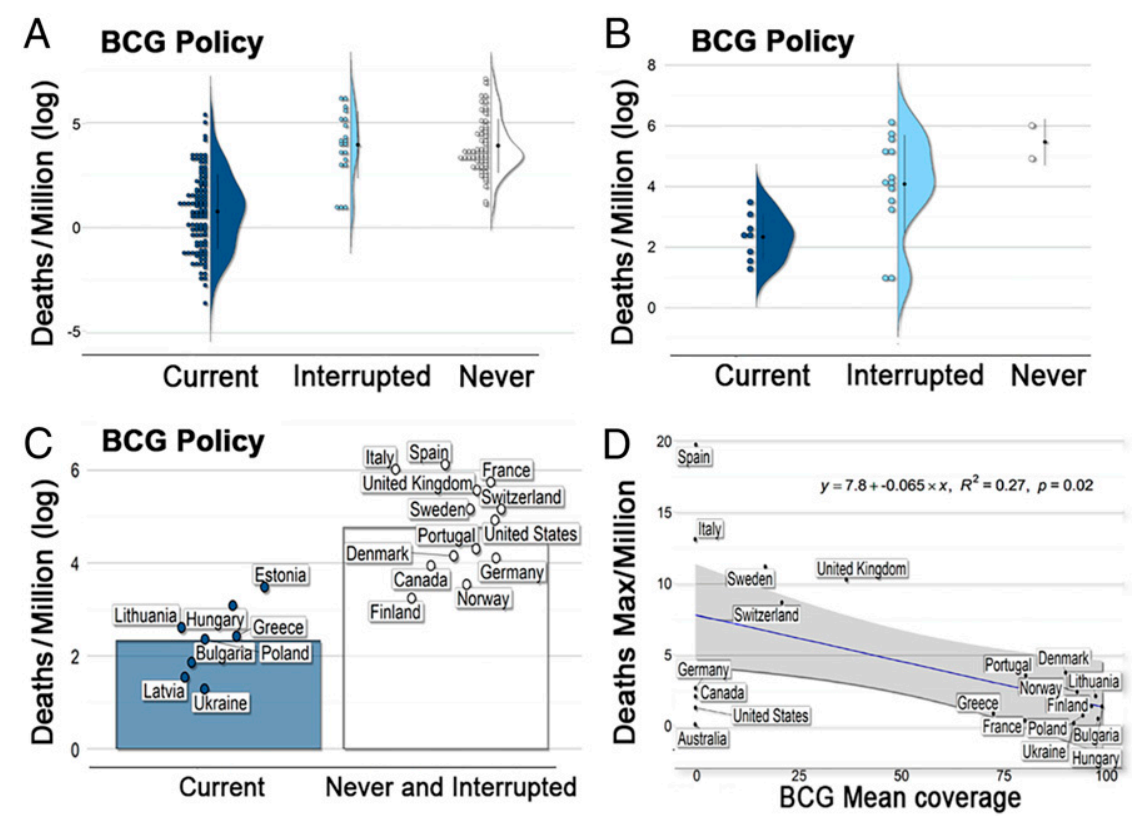

Fig. 2. Linkage between COVID-19 mortality and BCG vaccination. (A) Coarse analysis of COVID-19 mortality per million inhabitants in countries with current, interrupted, or that never had BCG vaccination programs. United States data appear by state. (B) Filtered analysis of COVID-19 mortality per million inhabitants in countries with current, interrupted, or that never had BCG vaccination programs and similar social, economic, and epidemic stage conditions. (C) Filtered analysis of COVID-19 mortality per million inhabitants in countries with current vs. interrupted or that never had BCG vaccination programs, including only countries with similar social, economic, and epidemic stage conditions. $(D)$ Negative association between percentage of vaccination coverage (mean) between 1980 and 2018, as a proxy of population protection, and maximum COVID-19 deaths per million inhabitants registered by country in a day, as a proxy of COVID-19 severity. Full list of analyses is available in SI Appendix, Tables S2-S4.

activation and resistance to M. tuberculosis (38). Enhanced susceptibility to TB is seen in humans with mutations in the $\gamma$-IFN receptor (38) and in mice in which the $\gamma$-IFN gene has been disrupted (39). The BCG vaccine is thought to confer protection from TB by enhancing cellular immunity.

The innate immune system is the first line of defense against invading pathogens. Immune cells constantly patrol the different organs, especially the gastrointestinal tract, airways, and the lungs, which have direct contact with the environment. For many decades, the innate immune response was thought to be hardwired and unable to adapt and "learn" from previous exposure to a pathogen. In the last $10 \mathrm{y}$, several studies demonstrated that the priming or "training" of the innate immune system is an ancient response observed in evolutionarily distant organisms, including plants (40), insects (41), and humans (42). Trained immunity, defined as the enhancement in innate immune responses to subsequent infections, is achieved through epigenetic and metabolic programming of immune cells that allows them to mount a stronger response to pathogens and to activate adaptive responses more efficiently (43). Trained immunity can confer broad protection that is not pathogen specific. For example, BCG vaccination is approved as a treatment for cancer of the bladder, and destruction of cancer cells has been shown to be mediated by trained immunity (44). Furthermore, BCG vaccination has been shown to elicit longlasting innate immune responses, beyond those specific to mycobacteria (45), and to modify hematopoietic stem cells, resulting in epigenetically modified macrophages that provide significantly better protection against virulent $M$. tuberculosis infection than naïve macrophages (46). Taken together, our current understanding of broad immune protection mediated by trained immunity and the epidemiologic evidence of longlasting protection from viral infections of the respiratory tract, conferred by BCG vaccination, offer a rational biological basis for the potential protective effect of $B C G$ vaccination from severe COVID-19. This response to BCG vaccination appears to be mediated by a mechanism different from the cellular immune response that confers protection from systemic TB in children (13).

BCG Vaccination and COVID-19 Mortality. The consistent association between BCG vaccination and reduced severity of COVID-19 observed in these and other epidemiological explorations is remarkable, but not sufficient to establish causality between BCG vaccination and protection from severe COVID-19. Randomized clinical trials, such as those ongoing in Holland (47) and Australia (48), in which health workers are administered either the BCG vaccine or a placebo saline injection, will determine the extent to which BCG vaccination in adults confers protection from COVID-19. There is limited information on the safety of administering $\mathrm{BCG}$ to senior persons, since $\mathrm{BCG}$ is a vaccine based on a live attenuated Mycobacterium that should not be administered to immunocompromised individuals (49). M. tuberculosis infection can remain latent for decades and reactivate in the elderly when a senescent immune system loses the ability to contain the infection (50). Nevertheless, a small study found that vaccination of adults $\geq 65 \mathrm{y}$ old with $\mathrm{BCG}$ prevented acute upper respiratory tract infections (51), and there is an active clinical trial vaccinating adults aged $>65 \mathrm{y}$ with $\mathrm{BCG}$ to boost immunity (52).

Most striking, COVID-19-related deaths are significantly higher in countries with higher quality of life (Fig. 1), contradicting the expectation of lower rates of mortality in countries with improved health care systems. Because of the differences in latitude, one can envision that differences in climate, such as ambient temperature, between states in the United States and South America could be responsible for the lower mortality in southern countries. However, one must consider that the average temperatures in the months of March to April are mild in Mexico City $\left(62.2^{\circ} \mathrm{F}\right.$ to $\left.64.6{ }^{\circ} \mathrm{F}\right)$, Sao Paulo $\left(73.0^{\circ} \mathrm{F}\right.$ to $\left.69.3^{\circ} \mathrm{F}\right)$, 


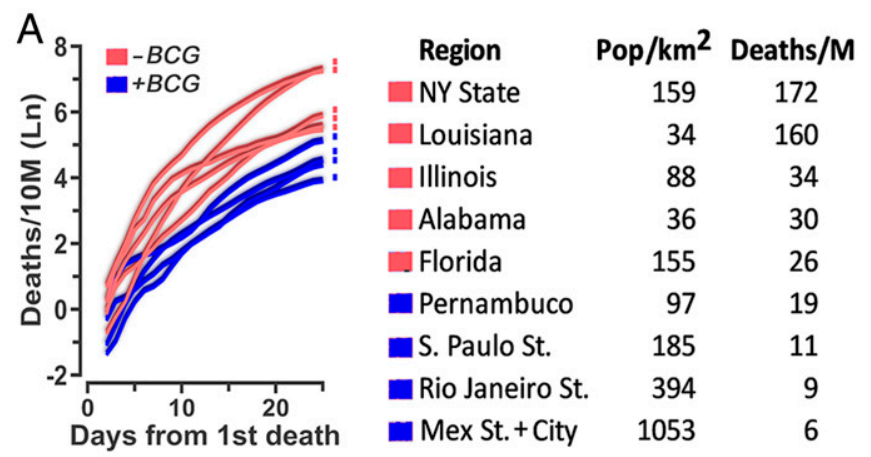

B
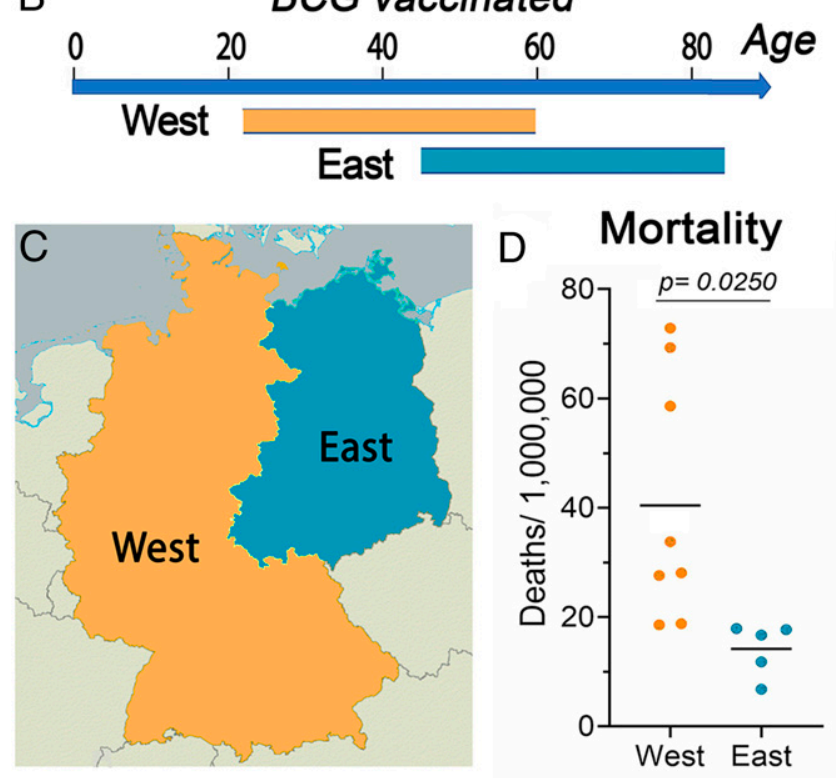

Fig. 3. COVID-19 mortality in comparable regions that have had different BCG vaccination policies. $(A)$ COVID-19 mortality by time in populous North and South American States. (Left) COVID-19 mortality per 10 million inhabitants in a 3-d centered average. Time adjusted according to the day with the first death in each region as day 1 , up to $25 \mathrm{~d}$ of the epidemic. (Right) Table shows population density and COVID-19 mortality by day 25 of the epidemic for each region. Regions that have had BCG vaccination (blue) had lower mortality than regions without BCG vaccination (red; $r^{2}=0.84, P<$ $0.001 ; t[237]=14.274, P<0.001)$. $(B)$ Estimated age range of people that received BCG vaccination in East and West Germany. (C) Map illustrating the regions of East and West Germany included in the analysis. (D) Mean COVID19 mortality in East Germany was lower than mortality in West Germany $(t[11]=-2.592, P=0.025)$.

Rio de Janeiro $\left(77.1^{\circ} \mathrm{F}\right.$ to $\left.73.9^{\circ} \mathrm{F}\right)$, Florida $\left(71.4^{\circ} \mathrm{F}\right.$ to $\left.74.8^{\circ} \mathrm{F}\right)$, Alabama $\left(60.1^{\circ} \mathrm{F}\right.$ to $\left.67.3^{\circ} \mathrm{F}\right)$, and Louisiana $\left(62.2^{\circ} \mathrm{F}\right.$ to $\left.69.3^{\circ} \mathrm{F}\right)$ (53), and that there is preliminary evidence for lack of temperature dependence for the COVID-19 epidemic (27, 28). The augmented COVID-19 mortality in developed countries when compared to developing countries remained consistent even after correcting for social confounding factors and age. There are several important social differences between eastern and western German states, such as lower GDP in former East Germany, and higher percentage of population $65 \mathrm{y}$ or older in eastern states $(24 \%)$ than in western ones $(21 \%)(34)$, but it is hard to envision how these factors could decrease their risk of COVID-19 fatality in eastern German states. One should consider that the difference in mortality could also be explained, at least in part, by the lower population density in East Germany $\left(154 / K_{\mathrm{m}}{ }^{2}\right)$ than in
West Germany $\left(282 / K_{\mathrm{m}}{ }^{2}\right)$. This variable is difficult to control, as there is great heterogeneity in population density even within each state.

The lack of apparent protection observed in the United Kingdom and France, where BCG vaccination was administered to older children, suggests that either trained immunity observed when infants are vaccinated is no longer achieved in older children or it may be of shorter duration. There may be a "critical
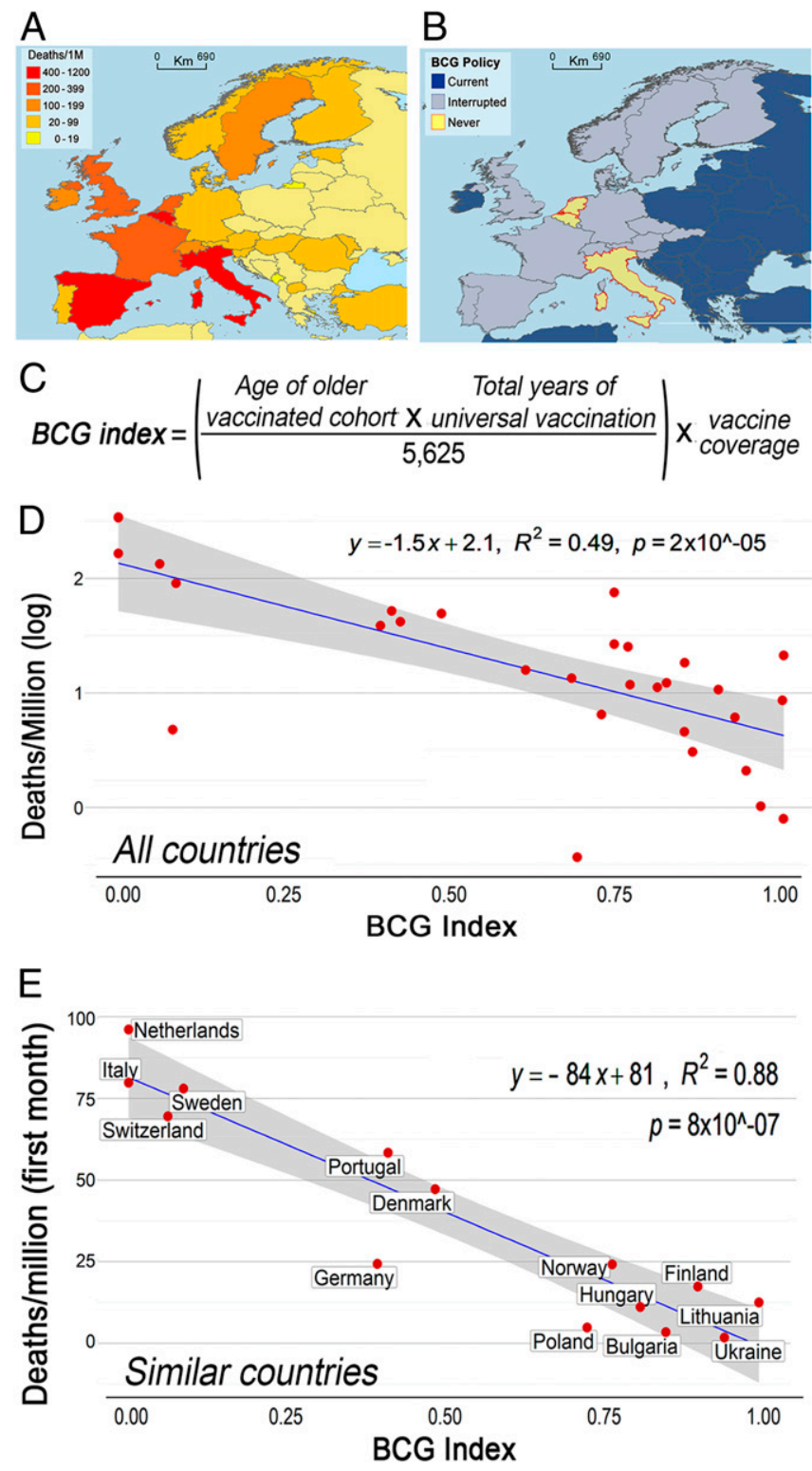

Fig. 4. Variation in COVID-19 mortality and BCG vaccination policy in different European countries. (A) Map illustrating COVID-19 deaths per million inhabitants in European countries. $(B)$ Map illustrating BCG vaccination policy in European countries that currently have universal BCG vaccination program (Current), countries that interrupted in the past a BCG vaccination program (Interrupted), and countries that never implemented a universal vaccination program (Never). (C) BCG index = (age of oldest vaccinated cohort $\times$ total number of years of vaccination campaign)/standardization parameter $(5,625) \times$ Mean BCG vaccination coverage. $(D)$ Correlation between BCG index and COVID-19 mortality per million inhabitants and in European countries with different BCG vaccination policy. $(E)$ Correlation between BCG index and COVID-19 mortality per million inhabitants during the first month of the pandemic in socially similar European countries with different BCG vaccination policy. 
window" early in life where BCG vaccination can result in lifelong enhanced immune surveillance. It is also possible that the BCG strains used, or the administration route, also affect the innate immune response to vaccination. Considering the remarkable dissemination capacity and the mortality rates of COVID-19, vaccination capable of conferring even transient protection (e.g., 6 mo to $12 \mathrm{mo}$ ) may be useful in individuals at high risk, such as health workers, first responders, and police officers, or those with preexisting conditions such as obesity, diabetes, or cardiovascular disease. Similarly, even enhanced unspecific immunity through BCG vaccination in vulnerable age groups could ameliorate severe COVID-19. Temporarily induced trained immunity could buy time until specific vaccines and/or effective treatments against severe acute respiratory syndrome coronavirus 2 infections become available.

If the BCG protection hypothesis holds true, it would have great implications for regions with ongoing universal vaccination programs, including most developing countries, as they may experience lower morbidity and mortality during the pandemic than in Europe and North America. We found that the number of years that universal BCG vaccination has been implemented in a given country and the level of vaccination coverage may play key roles in reducing of COVID-19 severity. For example, in many Latin American countries, universal vaccination was introduced in the mid-1960s, suggesting that individuals aged $\geq 55 \mathrm{y}$ would not be vaccinated and, in turn, represent a vulnerable segment of the population regarding COVID-19. Similarly, individuals born in years with low BCG vaccine coverage would be populations at risk. Most Asian countries have active universal BCG vaccination programs. If BCG is conferring some basal level of protection from COVID-19, it is possible that some of the social distancing roll-back strategies taken by Asian countries,

1. A. M. Abdallah, M. A. Behr, Evolution and strain variation in BCG. Adv. Exp. Med. Biol. 1019, 155-169 (2017).

2. C. Dye, Making wider use of the world's most widely used vaccine: Bacille CalmetteGuerin revaccination reconsidered. J. R. Soc. Interface 10, 20130365 (2013).

3. G. A. Colditz et al., The efficacy of bacillus Calmette-Guérin vaccination of newborns and infants in the prevention of tuberculosis: Meta-analyses of the published literature. Pediatrics 96, 29-35 (1995).

4. S. Luca, T. Mihaescu, History of BCG vaccine. Maedica (Buchar.) 8, 53-58 (2013).

5. P. Mangtani et al., Protection by BCG vaccine against tuberculosis: A systematic review of randomized controlled trials. Clin. Infect. Dis. 58, 470-480 (2014).

6. World Health Organization, BCG immunization coverage estimates by country. https://www.who.int/news-room/commentaries/detail/bacille-calmette-gu \% C3\% A9rin-(bcg)-vaccination-and-covid-19. Accessed 23 April 2020.

7. A. Zwerling et al., The BCG World Atlas: A database of global BCG vaccination policies and practices. PLoS Med. 8, e1001012 (2011).

8. C. Näslund, Resultats des experiences de vaccination par le BCG poursuivies dans le Norrbotten (Suède) (Septembre 1927-Décembre 1931). Vaccination Preventative de Tuberculose, Rapports et Documents, (Institut Pasteur, Paris, France, 1932).

9. M. L. Garly et al., BCG scar and positive tuberculin reaction associated with reduced child mortality in West Africa. A non-specific beneficial effect of BCG? Vaccine 21, 2782-2790 (2003).

10. P. Aaby et al., Randomized trial of BCG vaccination at birth to low-birth-weight children: Beneficial nonspecific effects in the neonatal period? J. Infect. Dis. 204, 245-252 (2011).

11. L. G. Stensballe et al., Acute lower respiratory tract infections and respiratory syncytial virus in infants in Guinea-Bissau: A beneficial effect of BCG vaccination for girls community based case-control study. Vaccine 23, 1251-1257 (2005).

12. M. J. de Castro, J. Pardo-Seco, F. Martinón-Torres, Nonspecific (heterologous) protection of neonatal BCG vaccination against hospitalization due to respiratory infection and sepsis. Clin. Infect. Dis. 60, 1611-1619 (2015).

13. L. A. J. O'Neill, M. G. Netea, BCG-induced trained immunity: Can it offer protection against COVID-19? Nat. Rev. Immunol. 20, 335-337 (2020).

14. I. K. Sharquie, BCG is a good immunotherapeutic agent for viral and autoimmune diseases: Is it a new weapon against coronavirus (COVID-19)? Electron J. Gen. Med. 17, em229 (2020).

15. J. P. Sfakianos, P. K. Hegarty, G. Giannarini, A. R. Dinardo, A. M. Kamat, COVID-19 and bacillus Calmette-Guérin: What is the link? Eur Assoc Urol 3, 259-261 (2020).

16. R. de Freitas E Silva, R. Pitzurra, What are the factors influencing the COVID-19 outbreak in Latin America? Travel Med. Infect. Dis. 35, 101667 (2020).

17. World Health Organization, Bacille Calmette-Guérin (BCG) vaccination and COVID-19. https://www.who.int/news-room/commentaries/detail/bacille-calmette-guérin-(bcg)vaccination-and-covid-19. Accessed 23 April 2020. in order to restart their economies, may not be effective in North America and western European countries, and could result in a second wave of infections.

Our understanding of the biology of innate immune training is in its infancy $(42,43)$. Little is known about the capacity of BCG vaccination to confer broad immune enhancement and the functional correlates of protection. Our inability to confirm the null hypothesis of no effect of BCG on COVID-19 mortality could be explained by an alternative hypothesis of crossprotection mediated by BCG vaccination. We note, however, that the data used in this epidemiological study have important sampling biases, and that the statistical signal detected at the country level may not explain COVID-19 mortality at the local level. The possibility that a single exposure to an attenuated pathogen during infancy could result in lifelong enhancement in immune surveillance is remarkable, but the available epidemiological data, in the absence of direct evidence from clinical trials, is not sufficient to recommend the use of BCG for the control and prevention of COVID-19 or other emerging infectious diseases.

Data Availability. All raw data were obtained from open sources and have been cited and deposited in Datasets S1.

ACKNOWLEDGMENTS. We gratefully acknowledge Asher Kantor and Mark Johnson for editorial assistance, and Mariana Castaneda-Guzman, Huijie Qiao, and Ana Beatriz Barletta Ferreira for assistance on data collection and analysis. We thank Dr. A. Townsend Peterson for his insightful comments and suggestions. This work was supported by the Intramural Research Program of the Division of Intramural Research Intramural Grant Z01Al000947, National Institute of Allergy and Infectious Diseases, NIH, and Virginia Polytechnic Institute and State University startup funds for L.E.E.

18. Y. Fang et al., Sensitivity of chest CT for COVID-19: Comparison to RT-PCR. Radiology, 10.1148/radiol.2020200432 (2020)

19. D. Romero-Alvarez, A. T. Peterson, L. E. Escobar, Surveillance fatigue (fatigatio vigilantiae) during epidemics. Rev. chilena Infectol. 34, 289-290 (2017).

20. J. Wu, A. McCann, J. Katz, E. Peltier, Tracking the true toll of the coronavirus outbreak. NY Times, 2 July 2020. https://www.nytimes.com/interactive/2020/04/21/world/ coronavirus-missing-deaths.html. Accessed 2 July 2020.

21. T. Lumley, Package 'leaps': Regression subset selection. https://cran.r-project.org/web/ packages/leaps/leaps.pdf. Accessed 15 April 2020

22. N. J. Gotelli, A. M. Ellison, A Primer of Ecological Statistics, (Sinauer Associates, Sunderland, MA, 2013).

23. Ministerio de Salud Pública del Ecuador, Evaluación de la Estrategia Nacional de Inmunizaciones Ecuador 2017. https://www.paho.org/ecu/index.php?option=com docman\&view=download\&category_slug=inmunizaciones\&alias=673-evaluacion-dela-estrategia-nacional-de-inmunizaciones-ecuador-2017\&ltemid=599. Accessed 15 April 2020

24. E. Freire Rodrigues et al., Local tuberculosis georeference: $A$ tool to define $B C G$ vaccination in high-incidence area in Portugal. Eur. J. Public Health 29, ckz186.532 (2019).

25. E. Dong, H. Du, L. Gardner, An interactive web-based dashboard to track COVID-19 in real time. Lancet Infect. Dis. 20, 533-534 (2020).

26. S. A. Lauer et al., The incubation period of coronavirus disease 2019 (COVID-19) from publicly reported confirmed cases: Estimation and application. Ann. Intern. Med. 172, 577-582 (2020).

27. T. Jamil, I. Alam, T. Gojobori, C. M. Duarte, No evidence for temperature-dependence of the COVID-19 epidemic. medRxiv:10.1101/2020.03.29.20046706 (19 April 2020).

28. M. T. P. Coelho, et al., Exponential phase of covid 19 expansion is not driven by climate at global scale. medRxiv:10.1101/2020.04.02.20050773 (6 May 2020)

29. United Nations Development Program, Human development data (1990-2018). Human Development Reports (2020). http://hdr.undp.org/en/data. Accessed 14 April 2020.

30. World Bank, World Development Indicators 2.1: Population dynamics. http://wdi. worldbank.org/table/2.1. Accessed 14 April 2020.

31. World Bank, Population density (people per sq. $\mathrm{km}$ of land area). https://data. worldbank.org/indicator/EN.POP.DNST. Accessed 14 April 2020.

32. World Bank, Urban population (\% of total population). https://data.worldbank.org/ indicator/SP.URB.TOTL.IN.ZS. Accessed 14 April 2020.

33. H. Lau et al., The association between international and domestic air traffic and the coronavirus (COVID-19) outbreak. J. Microbiol. Immunol. Infect. 53, 467-472 (2020).

34. Federal Statistical Office of Germany, Older people in Germany and the EU. https:// www.bmfsfj.de/blob/113952/83dbe067b083c7e8475309a88da89721/aeltere-menschen-in-deutschland-und-in-der-eu-englisch-data.pdf. Accessed 15 April 2020. 
35. Robert Koch Institute, SARS-CoV-2: Situation report - 14 Apr 2020. https://www.rki.de/ DE/Content/InfAZ/N/Neuartiges_Coronavirus/Situationsberichte/2020-04-14-en.pdf. Accessed 15 April 2020.

36. French Government, BCG vaccination law. https://beta.legifrance.gouv.fr/jorf/id/ JORFTEXT000000705041. Accessed 15 April 2020

37. J. Chan, J. Flynn, The immunological aspects of latency in tuberculosis. Clin. Immunol. 110, 2-12 (2004).

38. M. Rossouw, H. J. Nel, G. S. Cooke, P. D. van Helden, E. G. Hoal, Association between tuberculosis and a polymorphic NFkappaB binding site in the interferon gamma gene. Lancet 361, 1871-1872 (2003).

39. J. L. Flynn et al., An essential role for interferon gamma in resistance to Mycobac terium tuberculosis infection. J. Exp. Med. 178, 2249-2254 (1993)

40. M. Muthamilarasan, M. Prasad, Plant innate immunity: An updated insight into defense mechanism. J. Biosci. 38, 433-449 (2013)

41. J. Rodrigues, F. A. Brayner, L. C. Alves, R. Dixit, C. Barillas-Mury, Hemocyte differentiation mediates innate immune memory in Anopheles gambiae mosquitoes. Science 329, 1353-1355 (2010).

42. W. J. M. Mulder, J. Ochando, L. A. B. Joosten, Z. A. Fayad, M. G. Netea, Therapeutic targeting of trained immunity. Nat. Rev. Drug Discov. 18, 553-566 (2019).

43. M. G. Netea, E. Latz, K. H. Mills, L. A. O'Neill, Innate immune memory: A paradigm shift in understanding host defense. Nat. Immunol. 16, 675-679 (2015).

44. K. Buffen et al., Autophagy controls BCG-induced trained immunity and the response to intravesical BCG therapy for bladder cancer. PLoS Pathog. 10, e1004485 (2014).
45. J. Kleinnijenhuis et al., Long-lasting effects of BCG vaccination on both heterologous Th1/Th17 responses and innate trained immunity. J. Innate Immun. 6, 152-158 (2014).

46. E. Kaufmann et al., BCG educates hematopoietic stem cells to generate protective innate immunity against tuberculosis. Cell 172, 176-190.e19 (2018).

47. US National Library of Medicine, Reducing health care workers absenteeism in covid-19 pandemic through BCG vaccine (BCG-CORONA). ClinicalTrials.gov (2020). https://clinicaltrials.gov/ct2/show/NCT04328441. Accessed 15 April 2020.

48. US National Library of Medicine, BCG vaccination to protect healthcare workers against COVID-19 (BRACE). ClinicalTrials.gov (2020). https://clinicaltrials.gov/ct2/show/ NCT04327206. Accessed 15 April 2020.

49. A. C. Hesseling et al., The risk of disseminated Bacille Calmette-Guerin (BCG) disease in HIV-infected children. Vaccine 25, 14-18 (2007).

50. S. Rajagopalan, Tuberculosis and aging: A global health problem. Clin. Infect. Dis. 33, 1034-1039 (2001).

51. E. A. Wardhana, E. A. Datau, A. Sultana, V. V. Mandang, E. Jim, The efficacy of Bacillus Calmette-Guerin vaccinations for the prevention of acute upper respiratory tract infection in the elderly. Acta Med. Indones. 43, 185-190 (2011).

52. US National Library of Medicine, Bacillus Calmette-Guérin vaccination to prevent infections of the elderly (ACTIVATE). ClinicalTrials.gov (2017). https://clinicaltrials.gov/ ct2/show/record/NCT03296423. Accessed 14 April 2020.

53. Weather2Visit, Weather averages \& monthly temperatures. https://www.weather2visit.com. Accessed 31 May 2020. 